\title{
Advances in the Correlation between Intestinal Microbiota and Breast Cancer Development
}

\author{
Yonglan Jiang, Xinping Chen, Shengmiao Fu \\ Hainan Hospital Affiliated to The Hainan Medical College, Central Laboratory, Hainan General Hospital, Hainan Provincial Key \\ Laboratory of Cell and Molecular Genetic Translational Medicine, Hainan, China \\ Email: chenxinping@126.com,smfu2000@126.com
}

How to cite this paper: Jiang, Y.L., Chen, X.P. and Fu, S.M. (2020) Advances in the Correlation between Intestinal Microbiota and Breast Cancer Development. Journal of Cancer Therapy, 11, 758-771. https://doi.org/10.4236/jct.2020.1112066

Received: November 14, 2020

Accepted: December 8, 2020

Published: December 11, 2020

Copyright $\odot 2020$ by author(s) and Scientific Research Publishing Inc. This work is licensed under the Creative Commons Attribution International License (CC BY 4.0).

http://creativecommons.org/licenses/by/4.0/

\begin{abstract}
The intestinal microbiota has a symbiotic relationship with humans. It participates in some important physiological activities in the human body and has an important impact on human health. It has become a hot topic of research by scientists in recent years. Among them, the research on the correlation between intestinal microbiota and cancer has increased rapidly. At present, the incidence rate of breast cancer is increasing, which seriously endangers the health of women. More and more studies have found that the occurrence of breast cancer is related to intestinal microbiota, and its possible mechanism includes intestinal microbiota dysbiosis, estrogen metabolism changes, immune regulation, and the participation of intestinal microbiota metabolites, etc. With the further development of high-throughput sequencing technology, the research on the correlation between intestinal microbiota and breast cancer has become more in-depth, from a structural level confined to microorganisms to a more comprehensive system structure and function level. These research results provide a new research direction for the treatment of breast cancer. In order to further study the interaction between intestinal microbes and breast cancer, this article will comprehensively describe the intestinal microbiota and breast cancer from four aspects: intestinal microbial dysbiosis, altered estrogen metabolism, immune regulation, and intestinal microbial metabolites. It also reviews the application research of intestinal microbiota in breast cancer treatment, including the influence of intestinal microbiota on the effects of breast cancer radiotherapy and chemotherapy, probiotic therapy, and dietotherapy.
\end{abstract}

\section{Keywords}

Breast Cancer, Microbial Dysbiosis, Estrogen Metabolism, Immune Response, Microbial Metabolism 


\section{Introduction}

The intestinal microbiota is a large and diverse community of microorganisms designated to inhabit the human intestinal tract, consisting mainly of more than a thousand species of bacteria, but also fungi, viruses, archaea, and protozoa [1]. The gut microbiome, also known as the "second genome" of the human body [2], is the sum total of the genetic information of the gut microbiota and is extremely complex in its functions. The gut microbiota prevents pathogenic microorganisms from attacking the body and is important in host metabolism, immunity and hormone regulation [3] [4], promoting health but also contributing to the development of disease under certain conditions.

Breast cancer is the third most common cancer worldwide; it is also the leading cause of death from cancer among women worldwide in 2018 [5]. Breast cancer is a multifactorial disease, and proven risk factors include advanced age, early menarche, late menopause, age at first full-term delivery, low parity, infertility, hormone replacement therapy, family history of breast cancer, obesity, and type 2 diabetes, etc.; however, over $70 \%$ of women with breast cancer [6] do not carry any of these risk factors. Therefore, we consider that there are other risk factors for the development of breast cancer. Numerous studies in the past decade have shown how the gut microbiota influences the development of specific cancers in various organs, including breast cancer, through a series of complex mechanisms; human intestinal microbiota as a risk factor for breast cancer has received extensive attention in recent years, and more and more studies have reported the mechanism of interaction between gut microbiota and breast cancer, and research on the application of intestinal microbiota in the treatment of breast cancer has been increasing.

In this paper, we discuss the latest findings on the relationship between gut microbiota and breast cancer, including the association between the structural changes or disorders of intestinal flora and the development and prognosis of breast cancer, the mechanism by which gut microbiota acts on breast cancer through regulating immune response and influencing estrogen metabolism, and the association between gut microbial metabolites and breast cancer, in order to reveal the mechanism of breast cancer development in breast cancer patients and provide rationale and reference for the prevention and treatment of breast cancer.

\section{Breast Cancer and Altered Structure of Intestinal Microbiota}

Under normal conditions, the interactions between host and gut microbes will resist invasive pathogens and prevent tumorigenesis, whereas disruption of gut flora composition may put the body into a dysregulated state, and this alteration of the local microbial environment will lead to disease initiation and progression [7]. As early as 1990, Mineli EB et al. [8] found significant differences in the gut flora of premenopausal breast cancer patients compared to healthy women by 
observing and counting the fecal extract after incubation under the microscope. Using a mouse model, Buchta Rosean C [9] et al. showed that the inflammation that occurs in the mammary gland may be associated with a disturbance in the gut flora, which will run through the whole process of tumor occurrence and progression and cross talk with tumor tissues.

Goedert JJ and co-workers published a series of studies that reduced beta-diversity and alpha-diversity of the gut microbiota in breast cancer patients after adjustment for age, body mass index (BMI), and other factors [10]. A recent study in mice [11] also showed that the use of cephalosporins antibiotics (cephalexin) exacerbated the decrease in the community diversity of gut microbial and led to tumor formation, suggesting a causal relationship between antibiotic use and the incidence of breast cancer and further supporting the hypothesis of reduced intestinal bacterial diversity in breast cancer patients.

Contrary to these results, Chinese scholars Zhu J et al. found an increased diversity of gut microbiota in breast cancer patients compared with healthy controls, but there was little difference between premenopausal cases and controls, whereas postmenopausal women with breast cancer had higher species richness and species diversity than controls, and reported 14 potential microbial markers [12] that could be used to diagnose breast cancer in postmenopausal women.

It has also been suggested that gut flora may also be related to breast cancer stage. For example, the number of Blautia $s p$. in the feces of breast cancer patients increases with the increase in grades, and the total number of five bacteria including Bacteroidetes in stage II and III patients is higher [13]. Recent studies have also found that HER2- breast cancer patients have a higher alpha diversity of microbiota and a more unique bacterial composition than HER2+ breast cancer patients [14]. Fernandez MF [15] et al. also found that reduced numbers of Methylobacterium in breast cancer patients were associated with invasive-prone breast cancer.

These findings all suggest that gut microbiota can be used as a biomarker for the diagnosis and staging of breast cancer, but the exact role of the microbiota in the development and treatment of breast cancer still needs further study, and it remains unclear whether alterations in the microbiota cause the cancer or the cancer leads to alterations in the microbiota.

\section{Gut Microbiota Function and Breast Cancer}

There are various interactions between the human body and the intestinal microbiota. The host adjusts the composition of the microbiome in the body through its innate immune system in the intestine or its eating and living habits, but in turn, gut microbiota can also modulate the pathophysiological responses of the human body [16] [17]. For example, the function of the metabolites of the intestinal flora is like the hormones produced by the human body. They are synthesized and released by the intestinal microbes, absorbed into the blood circulation, and then transferred to other anatomical locations, where they exert 
their biological effects.

\subsection{Gut Microbiota Influences Estrogen Metabolism}

Estrogen has been shown to promote breast cancer and many studies have elucidated its mechanisms, including the induction of mitochondrial gene expression changes [18] and the promotion of breast epithelial cell and cancer cell proliferation [19]. Breast cancer risk is directly associated with higher endogenous estrogen levels and differences in estrogen metabolism, which is more significant in postmenopausal women [20] [21]. The association between gut microbes and estrogen has been proposed since the last century [22], and Plottel CS and Blaser MJ defined all the genes involved in estrogen metabolism in gut bacteria in 2011 [23], called "estrobolome" genes. Goedert and collaborators demonstrated that intestinal microbial diversity was positively associated with systemic, non-ovarian estrogens in both men and postmenopausal women, but not in premenopausal women [24] [25].

The intestinal microbiota is a key determinant of estrogen metabolism, and the intestinal flora is a major player in estrogen metabolism. Bacteria can uncouple the bound estrogens in the bile excreted into the intestine, and the uncoupling (or unbound) products are more easily reabsorbed into the bloodstream and enter the hepatic circulation, resulting in high levels of estrogenic substances in the body [26] [27]. While high estrogen levels have been mentioned above as a risk factor for breast cancer, the interaction of gut flora with estrogen in humans may be the pathway leading to the development and progression of breast cancer. Bacterial $\beta$-glucosidase is responsible for the uncoupling of estrogens, which is encoded by the GUS gene [28] [29] and the BG gene [30]. In recent years, studies have identified gastrointestinal microbial $\beta$-glucosidase profiles, which have been established so that we may be able to adjust the body's estrogen metabolism by altering the intestinal microbiota and further regulate the progression of breast cancer.

\subsection{Gut Microbiota and the Immune Response in Breast Cancer Patients}

Increased risk of breast cancer is associated with the presence of chronic, persistent and dysregulated inflammation [31] [32]. The intestinal mucosa has a huge surface area, colonized with a large number of microorganisms. These microbiotas interact with the host immune system and are involved in the development and functional regulation of the immune system, exerting an influence that cannot be ignored on the immune environment of the whole body, even the immune microenvironment [33] [34] [35] within the tumor. LAKRITZ JR and co-workers found that oral administration of $L$. reuteri reduced the risk of breast cancer in mice by stimulating the development of host $\mathrm{CD} 4^{+} \mathrm{CD} 25^{+} \mathrm{T}$ cells [36], leading to the conclusion that the intestinal flora can induce proliferation and differentiation of regulatory T cells. Peterson DA and collaborators demonstrat- 
ed that [37] gut microbiota can induce IgA protein expression through further studies. Goedert JJ finds only IgA-coated microbiota influence the risk of breast cancer through immune-mediated pathways, while non-IgA-coated microbiota does not [38]. Sun J and collaborators established a mouse model [39] in which the intestinal microflora was depleted by administration of broad-spectrum antibiotics, and the experimental results pointed out that intestinal microbiota regulates the immune environment by inducing the expression of antimicrobial peptides. In addition, dysbiosis of the intestinal flora will lead to a decrease in lymphocytes and an increase in neutrophils, both of which will be detrimental to the survival of breast cancer patients [40]. In turn, neutrophils, which are involved in the immune response to gut flora, also have a significant impact on breast cancer [41].

At the same time, there have been many studies that have shown that intestinal microbiota has an impact on the efficacy of immunotherapy in breast cancer patients. Therefore, regulating the intestinal flora to reduce the adverse effects of immunotherapy and increase the therapeutic effect will also become a kind of new combination therapy with strong applicability. Such as bactericides $s p$., especially $B$. thetaiotomicron and $B$. fragilis, can enhance the therapeutic effect of immunosuppressive(anti-CTLA-4 antibodies) [42]. On the other hand, some species of intestinal microbiota may upregulate the immune response by enhancing antigen presentation or increasing $\mathrm{T}$-cell recruitment in the local tumor environment [43]. Bifidobacterium and Acinetobacter mucinum have also been found to be associated with enhanced anti-PD-L1 therapeutic response [44] [45]. At present, the effect of gut microbiota on immunotherapy response of breast cancer patients is still unclear, and a foreign clinical trial (NCT02696759) is investigating whether gut microbiota can fight advanced breast cancer through immune modulation. It is also worth further studying to utilize probiotics to overcome the drug resistance and increase the efficacy of immunotherapy in some breast cancer patients.

\subsection{Gut Microbial Metabolites and Breast Cancer}

There is a complex and frequent communication between tumor tissue cells that strongly influences all aspects of tumorigenesis. At the same time, tumor cells interact systemically with the tumor and the entire body, including the intestinal microbiota. It has been found that the intestinal microbiota, through its metabolites, regulates all processes that promote tumor progression, including inflammation, angiogenesis, metabolism, and epithelial mesenchymal transition (EMT), acts on the tumor microenvironment(TME), and influences communication between the tumor microenvironment and the tumor and the entire organism [46]. Intestinal microorganisms produce a large number of metabolites, such as secondary metabolites, lipopolysaccharides, proteins, fermentation products, and breakdown metabolites, which can enter the circulation and interfere with the steady state of the intestine and areas away from the intestine, acting as sig- 
naling mediators to influence breast cancer progression [47].

\subsubsection{Short Chain Fatty Acids}

In breast cancer cells, short chain fatty acids (SCFAs) increase the expression of the adhesion protein E-cadherin by inhibiting the Hippo-yap pathway and inhibit mitogen-activated protein kinase (MAPK) signaling by binding free fatty acid receptor 3 (FFAR3), with the final result of reducing the invasive capacity of breast cancer cells and limiting metastasis formation by inducing EMT and regulating proliferative pathways [48]. SCFAs also inhibit histone deacetylase [49], prevent the expression of DNA repair proteins and compromise the integrity of DNA [50], so it can be said that SCFAs are regulators of invasion and apoptosis in breast cancer cells. In addition, studies have shown that intestinal alkaline phosphatase together with SCFAs enhance the tight junctions of the colonic mucosa and reduce the leakage of harmful pathogens and their carcinogenic potential [51] [52]. Among SCFAs, butyrate is mainly produced by firmicutes and acts as a protective agent for the colon epithelium, providing energy to the colon epithelial cells [53], while propionate may be metabolized by Bacteroidetes, but the role of propionate in breast cancer has been less studied so far.

\subsubsection{Lipopolysaccharides}

Lipopolysaccharide (LPS) can promote EMT, possibly by upregulating transforming growth factor $\beta-1$ (TGF $\beta-1$ ) and parental anti-epithelial homologues $2 / 3$ [54], and by promoting the expression of NF-KB and Toll-like receptor 4 (TLR4) in bile duct epithelial cells [55]. Lipopolysaccharide also activates vascular endothelial growth factor (VEGF) receptors and induces angiogenesis in tumor tissues [56]. Studies in breast cancer patients have also found that LPS stimulation of breast cancer cell lines MCF-7 and MDA-MB-231 induced cancer cell metastasis through activation of TLR4.

\subsubsection{Secondary Metabolites}

As early as 2002, deoxycholic acid (DCA) levels were found to be relatively high in the plasma of breast cyst fluid in postmenopausal breast cancer patients [57], suggesting that DCA can be involved in the carcinogenesis of organs that reach through the bloodstream, and it has subsequently been shown that DCA enhances the transcription of enzymes in cancer-associated fibroblasts [58]. On the contrary, the literature has shown that lithocholic acid (LCA) has an inhibitory function on breast cancer, especially by interacting with the bile acid receptor TGR5 [59].

\subsubsection{Cadaverine and Putrescine}

In addition, cadaverine (CAD) can be considered a tumor suppressor of breast cancer, which is derived from the decarboxylation of lysine by lysine decarboxylase. The biosynthesis of CAD is down-regulated in stage 1 breast cancer, which may be due to the reduction of gut microbiota that produces CAD in stage $1 \mathrm{pa}-$ tients, and higher levels of lysine decarboxylase have also been found in patients 
with higher survival rates. Cadaverine inhibits the EMT in breast cancer cells by activating trace amino acid receptors 8 and 9 (TAAR8/9) and regulating the expression of metalloproteinase 9 [60]. In addition, cadaverine can inhibit cell motility chemotaxis and metastasis to coordinate the occurrence of breast cancer. Another enzyme, ornithine decarboxylase(ODC), is responsible for the production of putrescine, which is upregulated in many cancers, and ODC is upregulated in many cancers, including breast cancer, and is associated with breast cancer progression, metastasis, and expression of estrogen receptor a [61], suggesting that putrescine may be a contributing factor to breast cancer.

\subsubsection{Catabolic Products}

Epidemiological studies have shown that high levels of phytoestrogens in the blood are negatively associated with breast cancer risk, unlike human endogenous estrogens [62] [63]. Intestinal bacteria can convert some plant lignans from flaxseed, sunflower seeds, beans, etc., into mammalian lignans such as enterolactone and enterodiol. Enterolactone may be a selective modulator of estrogen signaling and have protective effects against breast cancer [64] [65]. It has been suggested that regular intake of lignin-rich foods may reduce the risk of premenopausal breast cancer and increase the survival rate of postmenopausal women with breast cancer, leading some researchers to suggest that enterolactone may be a drug that can inhibit the proliferation of breast cancer cells [66]. However, a recent experimental study by Parida S et al. concluded that enterolactone is neither a drug for the treatment of breast cancer nor a risk factor for the development of breast cancer [67].

It can be seen that most of the metabolites of the intestinal microbiota are capable of activating different cellular receptors or have different positive or negative effects depending on the content and environment.

\section{Gut Microbiota and the Treatment of Breast Cancer}

\subsection{Gut Microbiota and Chemotherapy}

The absorption and bioavailability of most chemotherapeutic drugs require exposure to intestinal enzymes prior to their entry into the circulation, and the intestinal flora is the primary producers of these enzymes, which can alter the mechanism of action and toxicity of chemotherapeutic drugs [68] [69]. The gut microbiota has been shown to metabolize more than 40 drugs and may contribute to the therapeutic efficacy of many more [70]. Lehouritis $\mathrm{P}$ et al. tested the direct interaction of E. coli and Listeria welshimeri monocytogenes with 30 commonly used chemotherapeutic drugs in vitro and in vivo and found that one or two of the 10 drugs, whereas the efficacy of the six drugs was enhanced [71].

\subsection{Gut Microbiota and Radiotherapy}

Radiation therapy is another important treatment modality in the treatment of breast cancer. It has been shown that sterile and microbiota-controlled mice re- 
ceiving radiotherapy are less susceptible to dsDNA fragmentation in peripheral blood leukocytes than conventional mice [72], and that sterile mice are less susceptible to the toxic effects of cancer radiotherapy [73]. Thus, control of the microbiota by antibiotics can reduce the side effects of radiotherapy, improve patient compliance and improve patient outcomes.

\subsection{Probiotic Therapy}

The role of Lactobacillus in the treatment and prevention of breast cancer growth and metastasis has been confirmed by more and more experiments. Lakritz JR et al. showed that oral supplementation alone with purified L. reuteri was sufficient to inhibit early carcinogenesis and increase the sensitivity of breast cells to apoptosis in two groups of breast cancer mice fed a western diet and manipulated the development of genetic susceptibility [36]. In addition, oral administration of Lactobacillus acidophilus was shown to have anticancer activity in mice with mammary tumors [74]. Hassan Z et al. demonstrated that [75] Enterococcus faecalis and Staphylococcus hominis can significantly inhibit cell proliferation, induce apoptosis, and cell cycle arrest, and that they have no cytotoxic effect on normal cells, making them a good alternative drug for breast cancer treatment.

\subsection{Dietary Therapy}

Changing dietary patterns affects the microbiome and indirectly influences disease development. A case-control study in Japan showed that regular consumption of casein and soy isoflavones from puberty onwards reduced the incidence of breast cancer in Japanese women [76]; Newman TM et al. also indicated that the Mediterranean diet could prevent breast cancer [77]. Xue $\mathrm{M}$ et al. confirmed through experiments [78] that fucoidan increases the diversity of intestinal flora and can promote the intestinal barrier function, and he suggested fucoidan as a preventive agent for breast cancer.

These studies of gut flora in the treatment of breast cancer provide further evidence of the impact of gut flora on breast cancer and lay the groundwork for further research.

\section{Conclusions}

Breaking through the previous understanding of breast cancer, the intestinal flora plays a positive or negative role but non-negligible role in the progression of breast cancer. Although the importance of the gut microbiome in the development of breast cancer has been recognized, the mechanisms are not yet fully understood. On the one hand, it is due to the large and complex functioning of the gut microbiome; On the other hand, more than $90 \%$ of intestinal microbiota cannot be cultured or identified in vitro [1] [79]. The complex interactions between the gut microbiota are far beyond our understanding, and the pathophysiological effects of gut dysbiosis that we have identified so far are mostly the re- 
sult of the combined action of microbial groups rather than of individual microorganisms. Fortunately, the development of high-throughput sequencing technology has brought new breakthroughs in intestinal microbiome research, allowing us to explore more systematically and deeply the structure and function of gut microbes in breast cancer patients.

At present, the practical application of these research results is not ideal. Many researches are limited to mouse models, and a large number of clinical trials are still needed to translate these research results into clinical prevention and treatment of breast cancer patients. It is undeniable that research on the correlation between breast cancer and intestinal microbiota will eventually bring gospel to breast cancer patients. 1) Developing new breast cancer biomarkers, which is conducive to the early diagnosis of patients; 2) Good diet and lifestyle stabilize the intestinal flora, prevent the occurrence of breast cancer and tumor progression, improve the effect of radiotherapy and chemotherapy and prevent the side effects brought by radiotherapy and chemotherapy, and even prevent the recurrence of breast cancer, and improve the quality of life of patients; 3) New antitumor drugs may be developed according to the mechanism of intestinal microbiota promoting the progression of breast cancer.

\section{Conflicts of Interest}

The authors declare no conflicts of interest regarding the publication of this paper.

\section{References}

[1] Qin, J., Li, R., Raes, J., et al. (2010) A Human Gut Microbial Gene Catalogue Established by Metagenomic Sequencing. Nature, 464, 59-65.

https://doi.org/10.1038/nature08821

[2] Zhu, B., Wang, X. and Li, L. (2010) Human Gut Microbiome: The Second Genome of Human Body. Protein Cell, 1, 718-725. https://doi.org/10.1007/s13238-010-0093-Z

[3] Biragyn, A. and Ferrucci, L. (2018) Gut Dysbiosis: A Potential Link between Increased Cancer Risk in Ageing and Inflammaging. The Lancet Oncology, 19, e295-e304. https://doi.org/10.1016/S1470-2045(18)30095-0

[4] Maynard, C.L., Elson, C.O., Hatton, R.D., et al. (2012) Reciprocal Interactions of the Intestinal Microbiota and Immune System. Nature, 489, 231-241. https://doi.org/10.1038/nature11551

[5] Bray, F., Ferlay, J., Soerjomataram, I., et al. (2018) Global Cancer Statistics 2018: GLOBOCAN Estimates of Incidence and Mortality Worldwide for 36 Cancers in 185 Countries. CA: A Cancer Journal for Clinicians, 68, 394-424. https://doi.org/10.3322/caac.21492

[6] Lacey, J.V., Kreimer, A.R., Buys, S.S., et al. (2009) Breast Cancer Epidemiology According to Recognized Breast Cancer Risk Factors in the Prostate, Lung, Colorectal and Ovarian (PLCO) Cancer Screening Trial Cohort. BMC Cancer, 9, 84. https://doi.org/10.1186/1471-2407-9-84

[7] Shapira, I., Sultan, K., Lee, A., et al. (2013) Evolving Concepts: How Diet and the Intestinal Microbiome Act as Modulators of Breast Malignancy. ISRN Oncology, 
2013, Article ID: 693920. https://doi.org/10.1155/2013/693920

[8] Mineli, E.B., Beghini, A.M., Vesentini, S., et al. (1990) Intestinal Microflora as an Alternative Metabolic Source of Estrogens in Women with Uterine Leiomyoma and Breast Cancer. Annals of the New York Academy of Sciences, 595, 473-479. https://doi.org/10.1111/j.1749-6632.1990.tb34337.x

[9] Buchta Rosean, C., Bostic, R.R., Ferey, J.C.M., et al. (2019) Preexisting Commensal Dysbiosis Is a Host-Intrinsic Regulator of Tissue Inflammation and Tumor Cell Dissemination in Hormone Receptor-Positive Breast Cancer. Cancer Research, 79, 3662-3675. https://doi.org/10.1158/0008-5472.CAN-18-3464

[10] Goedert, J.J., Jones, G., Hua, X., et al. (2015) Investigation of the Association between the Fecal Microbiota and Breast Cancer in Postmenopausal Women: A Population-Based Case-Control Pilot Study. Journal of the National Cancer Institute, 107, djv147. https://doi.org/10.1093/jnci/djv147

[11] Kirkup, B., McKee, A., Makin, K., et al. (2019) Perturbation of the Gut Microbiota by Antibiotics Results in Accelerated Breast Tumour Growth and Metabolic Dysregulation. https://doi.org/10.1101/553602

[12] Zhu, J., Liao, M., Yao, Z., et al. (2018) Breast Cancer in Postmenopausal Women Is Associated with an Altered Gut Metagenome. Microbiome, 6, 136.

https://doi.org/10.1186/s40168-018-0515-3

[13] Luu, T.H., Michel, C., Bard, J.M., et al. (2017) Intestinal Proportion of Blautia sp. Is Associated with Clinical Stage and Histoprognostic Grade in Patients with Early-Stage Breast Cancer. Nutrition and Cancer, 69, 267-275. https://doi.org/10.1080/01635581.2017.1263750

[14] Wu, A.H., Tseng, C., Vigen, C., et al. (2020) Gut Microbiome Associations with Breast Cancer Risk Factors and Tumor Characteristics: A Pilot Study. Breast Cancer Research and Treatment, 182, 451-463. https://doi.org/10.1007/s10549-020-05702-6

[15] Fernández, M.F., Iris, R.-P., Manuel, A.J., et al. (2018) Breast Cancer and Its Relationship with the Microbiota. International Journal of Environmental Research and Public Health, 15, 1747. https://doi.org/10.3390/ijerph15081747

[16] Mikó, E., Vida, A. and Bai, P. (2016) Translational Aspects of the Microbiome-To Be Exploited. Cell Biology and Toxicology, 32, 153-156. https://doi.org/10.1007/s10565-016-9320-6

[17] Tao, J., Li, S., Gan, R.Y., et al. (2020) Targeting Gut Microbiota with Dietary Components on Cancer: Effects and Potential Mechanisms of Action. Critical Reviews in Food Science and Nutrition, 60, 1025-1037. https://doi.org/10.1080/10408398.2018.1555789

[18] Chen, J.Q. and Yager, J.D. (2004) Estrogen's Effects on Mitochondrial Gene Expression: Mechanisms and Potential Contributions to Estrogen Carcinogenesis. Annals of the New York Academy of Sciences, 1028, 258-272. https://doi.org/10.1196/annals.1322.030

[19] Samavat, H. and Kurzer, M.S. (2015) Estrogen Metabolism and Breast Cancer. Cancer Letters, 356, 231-243. https://doi.org/10.1016/j.canlet.2014.04.018

[20] Key, T.J., Appleby, P.N., Reeves, G.K., et al. (2003) Body Mass Index, Serum Sex Hormones, and Breast Cancer Risk in Postmenopausal Women. Journal of the National Cancer Institute, 95, 1218-1226. https://doi.org/10.1093/jnci/djg022

[21] Sampson, J.N., Falk, R.T., Schairer, C., et al. (2017) Association of Estrogen Metabolism with Breast Cancer Risk in Different Cohorts of Postmenopausal Women. Cancer Research, 77, 918-925. https://doi.org/10.1158/0008-5472.CAN-16-1717

[22] Adlercreutz, H., Pulkkinen, M.O., Hämäläinen, E.K., et al. (1984) Studies on the 
Role of Intestinal Bacteria in Metabolism of Synthetic and Natural Steroid Hormones. Journal of Steroid Biochemistry, 20, 217-229. https://doi.org/10.1016/0022-4731(84)90208-5

[23] Plottel, C.S. and Blaser, M.J. (2011) Microbiome and Malignancy. Cell Host \& Microbe, 10, 324-335. https://doi.org/10.1016/j.chom.2011.10.003

[24] Flores, R., Shi, J., Fuhrman, B., et al. (2012) Fecal Microbial Determinants of Fecal and Systemic Estrogens and Estrogen Metabolites: A Cross-Sectional Study. Journal of Translational Medicine, 10, Article No. 253. https://doi.org/10.1186/1479-5876-10-253

[25] Fuhrman, B.J., Feigelson, H.S., Flores, R., et al. (2014) Associations of the Fecal Microbiome with Urinary Estrogens and Estrogen Metabolites in Postmenopausal Women. The Journal of Clinical Endocrinology and Metabolism, 99, 4632-4640. https://doi.org/10.1210/jc.2014-2222

[26] Baker, J.M., Al-Nakkash, L. and Herbst-Kralovetz, M.M. (2017) Estrogen-Gut Microbiome Axis: Physiological and Clinical Implications. Maturitas, 103, 45-53. https://doi.org/10.1016/j.maturitas.2017.06.025

[27] Yang, J., Tan, Q., Fu, Q., et al. (2017) Gastrointestinal Microbiome and Breast Cancer: Correlations, Mechanisms and Potential Clinical Implications. Breast Cancer, 24, 220-228. https://doi.org/10.1007/s12282-016-0734-Z

[28] Dabek, M., McCrae, S.I., Stevens, V.J., et al. (2008) Distribution of Beta-Glucosidase and Beta-Glucuronidase Activity and of Beta-Glucuronidase Gene Gus in Human Colonic Bacteria. FEMS Microbiology Ecology, 66, 487-495.

https://doi.org/10.1111/j.1574-6941.2008.00520.x

[29] McIntosh, F.M., Maison, N., Holtrop, G., et al. (2012) Phylogenetic Distribution of Genes Encoding $\beta$-Glucuronidase Activity in Human Colonic Bacteria and the Impact of Diet on Faecal Glycosidase Activities. Environmental Microbiology, 14, 1876-1887. https://doi.org/10.1111/j.1462-2920.2012.02711.x

[30] Gloux, K., Berteau, O., El Oumami, H., et al. (2011) A Metagenomic $\beta$-Glucuronidase Uncovers a Core Adaptive Function of the Human Intestinal Microbiome. Proceedings of the National Academy of Sciences of the United States of America, 108, 4539-4546. https://doi.org/10.1073/pnas.1000066107

[31] Crusz, S.M. and Balkwill, F.R. (2015) Inflammation and Cancer: Advances and New Agents. Nature Reviews Clinical Oncology, 12, 584-596.

https://doi.org/10.1038/nrclinonc.2015.105

[32] Vogtmann, E. and Goedert, J.J. (2016) Epidemiologic Studies of the Human Microbiome and Cancer. British Journal of Cancer, 114, 237-242. https://doi.org/10.1038/bjc.2015.465

[33] Chassaing, B., Kumar, M., Baker, M.T., et al. (2014) Mammalian Gut Immunity. Biomedical Journal, 37, 246-258. https://doi.org/10.4103/2319-4170.130922

[34] Buchta Rosean, C.M. and Rutkowski, M.R. (2017) The Influence of the Commensal Microbiota on Distal Tumor-Promoting Inflammation. Seminars in Immunology, 32, 62-73. https://doi.org/10.1016/j.smim.2017.06.002

[35] Xuan, C., Shamonki, J.M., Chung, A., et al. (2014) Microbial Dysbiosis Is Associated with Human Breast Cancer. PLoS ONE, 9, e83744. https://doi.org/10.1371/journal.pone.0083744

[36] Lakritz, J.R., Poutahidis, T., Levkovich, T., et al. (2014) Beneficial Bacteria Stimulate Host Immune Cells to Counteract Dietary and Genetic Predisposition to Mammary Cancer in Mice. International Journal of Cancer, 135, 529-540. https://doi.org/10.1002/ijc.28702 
[37] Peterson, D.A., McNulty, N.P., Guruge, J.L., et al. (2007) IgA Response to Symbiotic Bacteria as a Mediator of Gut Homeostasis. Cell Host \& Microbe, 2, 328-339. https://doi.org/10.1016/j.chom.2007.09.013

[38] Goedert, J.J., Hua, X., Bielecka, A., et al. (2018) Postmenopausal Breast Cancer and Oestrogen Associations with the IgA-Coated and IgA-Noncoated Faecal Microbiota. British Journal of Cancer, 118, 471-479. https://doi.org/10.1038/bjc.2017.435

[39] Sun, J., Furio, L., Mecheri, R., et al. (2015) Pancreatic $\beta$-Cells Limit Autoimmune Diabetes via an Immunoregulatory Antimicrobial Peptide Expressed under the Influence of the Gut Microbiota. Immunity, 43, 304-317. https://doi.org/10.1016/j.immuni.2015.07.013

[40] Chace, D. (2015) Turning Off Breast Cancer: A Personalized Approach to Nutrition and Detoxification in Prevention and Healing. Skyhorse Publishing, Inc., New York.

[41] Rao, V.P., Poutahidis, T., Ge, Z., et al. (2006) Innate Immune Inflammatory Response against Enteric Bacteria Helicobacter hepaticus Induces Mammary Adenocarcinoma in Mice. Cancer Research, 66, 7395-7400. https://doi.org/10.1158/0008-5472.CAN-06-0558

[42] Vétizou, M., Pitt, J.M., Daillère, R., et al. (2015) Anticancer Immunotherapy by CTLA-4 Blockade Relies on the Gut Microbiota. Science, 350, 1079-1084. https://doi.org/10.1126/science.aad1329

[43] Gopalakrishnan, V., Spencer, C.N., Nezi, L., et al. (2018) Gut Microbiome Modulates Response to Anti-PD-1 Immunotherapy in Melanoma Patients. Science, 359, 97-103. https://doi.org/10.1126/science.aan4236

[44] Sivan, A., Corrales, L., Hubert, N., et al. (2015) Commensal Bifidobacterium Promotes Antitumor Immunity and Facilitates Anti-PD-L1 Efficacy. Science, 350, 1084-1089. https://doi.org/10.1126/science.aac4255

[45] Routy, B., Le Chatelier, E., Derosa, L., et al. (2018) Gut Microbiome Influences Efficacy of PD-1-Based Immunotherapy against Epithelial Tumors. Science, 359, 91-97. https://doi.org/10.1126/science.aan3706

[46] Rossi, T., Vergara, D., Fanini, F., et al. (2020) Microbiota-Derived Metabolites in Tumor Progression and Metastasis. International Journal of Molecular Sciences, 21, 5786. https://doi.org/10.3390/ijms21165786

[47] Mikó, E., Kovács, T., Sebő, É., et al. (2019) Microbiome-Microbial Metabolome-Cancer Cell Interactions in Breast Cancer-Familiar, but Unexplored. Cells, 8, 293. https://doi.org/10.3390/cells8040293

[48] Thirunavukkarasan, M., Wang, C., Rao, A., et al. (2017) Short-Chain Fatty Acid Receptors Inhibit Invasive Phenotypes in Breast Cancer Cells. PLoS ONE, 12, e0186334. https://doi.org/10.1371/journal.pone.0186334

[49] Kiweler, N., Wünsch, D., Wirth, M., et al. (2020) Histone Deacetylase Inhibitors Dysregulate DNA Repair Proteins and Antagonize Metastasis-Associated Processes. Journal of Cancer Research and Clinical Oncology, 146, 343-356. https://doi.org/10.1007/s00432-019-03118-4

[50] Salimi, V., Shahsavari, Z., Safizadeh, B., et al. (2017) Sodium Butyrate Promotes Apoptosis in Breast Cancer Cells through Reactive Oxygen Species (ROS) Formation and Mitochondrial Impairment. Lipids in Health and Disease, 16, Article No. 208. https://doi.org/10.1186/s12944-017-0593-4

[51] Ashida, H., Ogawa, M., Kim, M., et al. (2011) Bacteria and Host Interactions in the Gut Epithelial Barrier. Nature Chemical Biology, 8, 36-45.

https://doi.org/10.1038/nchembio.741 
[52] Rubin, R. (2019) High-Fiber Diet Might Protect against Range of Conditions. JAMA, 321, 1653-1655. https://doi.org/10.1001/jama.2019.2539

[53] Vital, M., Howe, A.C. and Tiedje, J.M. (2014) Revealing the Bacterial Butyrate Synthesis Pathways by Analyzing (Meta)genomic Data. mBio, 5, e00889.

https://doi.org/10.1128/mBio.00889-14

[54] Zhao, L., Yang, R., Cheng, L., et al. (2011) LPS-Induced Epithelial-Mesenchymal Transition of Intrahepatic Biliary Epithelial Cells. Journal of Surgical Research, 171, 819-825. https://doi.org/10.1016/j.jss.2010.04.059

[55] Kim, Y., Lee, E.J., Jang, H.K., et al. (2016) Statin Pretreatment Inhibits the Lipopolysaccharide-Induced Epithelial-Mesenchymal Transition via the Downregulation of Toll-Like Receptor 4 and Nuclear Factor- $\kappa$ B in Human Biliary Epithelial Cells. Journal of Gastroenterology and Hepatology, 31, 1220-1228.

https://doi.org/10.1111/jgh.13230

[56] Zhu, G.H.Q., Huang, Y., Zheng, W., Hua, J., Yang, S., Zhuang, J., Wang, J. and Ye, J. (2016) Lipopolysaccharide Increases the Release of VEGF-C That Enhances Cell Motility and Promotes Lymphangiogenesis and Lymphatic Metastasis through the TLR4-NF-kB/JNK Pathways in Colorectal Cancer. Oncotarget, 7, 73711-73724. https://doi.org/10.18632/oncotarget.12449

[57] Costarelli, V. and Sanders, T.A. (2002) Plasma Deoxycholic Acid Concentration Is Elevated in Postmenopausal Women with Newly Diagnosed Breast Cancer. European Journal of Clinical Nutrition, 56, 925-927. https://doi.org/10.1038/sj.ejcn.1601396

[58] Zhu, Y., Zhu, M. and Lance, P. (2012) Stromal COX-2 Signaling Activated by Deoxycholic Acid Mediates Proliferation and Invasiveness of Colorectal Epithelial Cancer Cells. Biochemical and Biophysical Research Communications, 425, 607-612. https://doi.org/10.1016/j.bbrc.2012.07.137

[59] Mikó, E., Vida, A., Kovács, T., et al. (2018) Lithocholic Acid, a Bacterial Metabolite Reduces Breast Cancer Cell Proliferation and Aggressiveness. Biochimica et Biophysica Acta-Bioenergetics, 1859, 958-974. https://doi.org/10.1016/j.bbabio.2018.04.002

[60] Kovács, T., Mikó, E., Vida, A., et al. (2019) Cadaverine, a Metabolite of the Microbiome, Reduces Breast Cancer Aggressiveness through Trace Amino Acid Receptors. Scientific Reports, 9, Article No. 1300. https://doi.org/10.1038/s41598-018-37664-7

[61] Zhu, Q., Jin, L., Casero, R.A., et al. (2012) Role of Ornithine Decarboxylase in Regulation of Estrogen Receptor Alpha Expression and Growth in Human Breast Cancer Cells. Breast Cancer Research and Treatment, 136, 57-66. https://doi.org/10.1007/s10549-012-2235-x

[62] Ward, H.A. and Kuhnle, G.G. (2010) Phytoestrogen Consumption and Association with Breast, Prostate and Colorectal Cancer in EPIC Norfolk. Archives of Biochemistry and Biophysics, 501, 170-175. https://doi.org/10.1016/j.abb.2010.05.018

[63] Milder, I.E., Kuijsten, A., Arts, I.C., et al. (2007) Relation between Plasma Enterodiol and Enterolactone and Dietary Intake of Lignans in a Dutch Endoscopy-Based Population. Journal of Nutrition, 137, 1266-1271.

https://doi.org/10.1093/jn/137.5.1266

[64] Pietinen, P., Stumpf, K., Männistö, S., et al. (2001) Serum Enterolactone and Risk of Breast Cancer: A Case-Control Study in Eastern Finland. Cancer Epidemiology, Biomarkers \& Prevention, 10, 339-344.

[65] Saarinen, N.M., Wärri, A., Airio, M., et al. (2007) Role of Dietary Lignans in the 
Reduction of Breast Cancer Risk. Molecular Nutrition \& Food Research, 51, 857-866. https://doi.org/10.1002/mnfr.200600240

[66] Cotterchio, M., Boucher, B.A., Kreiger, N., et al. (2008) Dietary Phytoestrogen Intake-Lignans and Isoflavones-And Breast Cancer Risk (Canada). Cancer Causes \& Control, 19, 259-272. https://doi.org/10.1007/s10552-007-9089-2

[67] Parida, S. and Sharma, D. (2019) The Microbiome-Estrogen Connection and Breast Cancer Risk. Cells, 8, 1642. https://doi.org/10.3390/cells8121642

[68] Roy, S. and Trinchieri, G. (2017) Microbiota: A Key Orchestrator of Cancer Therapy. Nature Reviews Cancer, 17, 271-285. https://doi.org/10.1038/nrc.2017.13

[69] Alexander, J.L., Wilson, I.D., Teare, J., et al. (2017) Gut Microbiota Modulation of Chemotherapy Efficacy and Toxicity. Nature Reviews Gastroenterology \& Hepatology, 14, 356-365. https://doi.org/10.1038/nrgastro.2017.20

[70] Haiser, H.J. and Turnbaugh, P.J. (2013) Developing a Metagenomic View of Xenobiotic Metabolism. Pharmacological Research, 69, 21-31.

https://doi.org/10.1016/j.phrs.2012.07.009

[71] Lehouritis, P., Cummins, J., Stanton, M., et al. (2015) Local Bacteria Affect the Efficacy of Chemotherapeutic Drugs. Scientific Reports, 5, Article No. 14554. https://doi.org/10.1038/srep14554

[72] Maier, I., Berry, D.M. and Schiestl, R.H. (2014) Intestinal Microbiota Reduces Genotoxic Endpoints Induced by High-Energy Protons. Radiation Research, 181, 45-53. https://doi.org/10.1667/RR13352.1

[73] Touchefeu, Y., Montassier, E., Nieman, K., et al. (2014) Systematic Review: The Role of the Gut Microbiota in Chemotherapy- or Radiation-Induced Gastrointestinal Mucositis-Current Evidence and Potential Clinical Applications. Alimentary Pharmacology \& Therapeutics, 40, 409-421. https://doi.org/10.1111/apt.12878

[74] Yazdi, M.H., Soltan Dallal, M.M., Hassan, Z.M., et al. (2010) Oral Administration of Lactobacillus acidophilus Induces IL-12 Production in Spleen Cell Culture of BALB/c Mice Bearing Transplanted Breast Tumour. British Journal of Nutrition, 104, 227-232. https://doi.org/10.1017/S0007114510000516

[75] Hassan, Z., Mustafa, S., Rahim, R.A., et al. (2016) Anti-Breast Cancer Effects of Live, Heat-Killed and Cytoplasmic Fractions of Enterococcus faecalis and Staphylococcus hominis Isolated from Human Breast Milk. In Vitro Cellular \& Developmental Biology_Animal, 52, 337-348. https://doi.org/10.1007/s11626-015-9978-8

[76] Toi, M., Hirota, S., Tomotaki, A., et al. (2013) Probiotic Beverage with Soy Isoflavone Consumption for Breast Cancer Prevention: A Case-Control Study. Current Nutrition \& Food Science, 9, 194-200. https://doi.org/10.2174/15734013113099990001

[77] Newman, T.M., Vitolins, M.Z. and Cook, K.L. (2019) From the Table to the Tumor: The Role of Mediterranean and Western Dietary Patterns in Shifting Microbial-Mediated Signaling to Impact Breast Cancer Risk. Nutrients, 11, 2565.

https://doi.org/10.3390/nu11112565

[78] Xue, M., Ji, X., Liang, H., et al. (2018) The Effect of Fucoidan on Intestinal Flora and Intestinal Barrier Function in Rats with Breast Cancer. Food \& Function, 9, 1214-1223. https://doi.org/10.1039/C7FO01677H

[79] Hullar, M.A., Burnett-Hartman, A.N. and Lampe, J.W. (2014) Gut Microbes, Diet, and Cancer. Cancer Treatment and Research, 159, 377-399.

https://doi.org/10.1007/978-3-642-38007-5 22 